\title{
Reduced adhesion of human blood platelets to polyethylene tubing by microplasma surface modification
}

\author{
J. L. Lauer, J. L. Shohet, R. M. Albrecht, C. Pratoomtong, R. Murugesan, S. Esnault, \\ J. S. Malter, U. H. von Andrian, ${ }^{\text {a) }}$ R. D. Bathke, and S. B. Shohet ${ }^{\text {b) }}$ \\ Plasma Processing and Technology Laboratory and Department of Electrical \& Computer Engineering, \\ University of Wisconsin-Madison, Madison, Wisconsin 53706
}

(Received 12 March 2004; accepted 5 July 2004)

\begin{abstract}
A hollow-cathode microplasma modified the lumenal surface of small-diameter polyethylene (PE) tubing. A microwave cavity diagnostic was used to measure the density of the microplasma. Plasma light output was observed with a monochromator at various positions along the PE tube to assess uniformity. Treatment effectiveness was evaluated by measuring the variation in capillary rise at various positions along the tubing. A correlation between the properties of the inner surface of the PE tubing and the emitted light intensity was found. A poly(ethylene oxide) surfactant was immobilized to the lumenal surface of the PE tubing with an argon microplasma discharge. To test hematocompatibility, an in vitro blood-flow loop circulated heparinized human blood through both a plasma-treated and -untreated PE tubes, simultaneously. After blood exposure, the tubes were examined with a scanning electron microscope to assess the density of adhering platelets along the length of the tubes. By modifying the plasma parameters, the uniformity of the microplasma treatment along the tubing can be optimized. (C) 2004 American Institute of Physics.
\end{abstract}

[DOI: $10.1063 / 1.1786668]$

\section{INTRODUCTION}

The use of microplasmas for the surface treatment of small diameter $(<2 \mathrm{~mm})$ polymer tubing will provide practical uses of plasmas in the area of biotechnology and for the use of polymer tubing ${ }^{1,2}$ in general. To date, most studies of plasma-modified biomaterials make use of a large-volume plasma reactors to modify the surface of a small disk or film substrate. ${ }^{3-6}$ However, such reactors are not very practical for surface modification of small diameter tubing ${ }^{1}$ and many small-scale biomedical devices. ${ }^{9}$ This is because the plasma tends to quench itself near the ends of the tubing, ${ }^{7,8}$ when placed in a large-volume reactor, and thus only small portions near the ends of the tube are treated. ${ }^{1}$ Also, complications in treating the side walls of narrow trenches and reservoirs of small-scale biomedical devices can cause nonuniformity of the coating. This is because the flux of energetic plasma species to the bottom of trenches will not be the same as to the sidewalls, thus producing further nonuniform plasma treatment.

The realization of microfluidic devices that are in contact with various cells, proteins, and a range of $p \mathrm{H}$ values will be closely related to the nonfouling/biocompatible properties of the exposed surfaces and the uniformity of the surface treatment throughout the devices. By choosing the proper frequency, electrode configuration, gas inlet type, and flow rate, microplasma surface modification has the potential to treat surfaces of a variety of shapes and sizes that are contained in the small volume of a biomedical device. This could be done by embedding ${ }^{9}$ microelectrodes into the structure of the device itself. This would allow for a different surface-specific

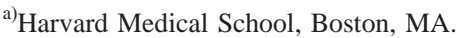

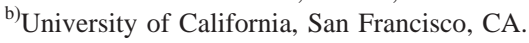

treatment of various chambers and channels throughout a device. This could be done during fabrication or after the device is built, by connecting the leads of the electrodes to the appropriate excitation source (microwave, ${ }^{10-12}$ radiofrequency, ${ }^{13,14}$ low frequency, ${ }^{15,16}$ or $\mathrm{dc}^{17-19}$ ) and inletting the proper ${ }^{6}$ gas monomer(s) or surfactant coating. Also, by proper placement of the microelectrodes within the device, it may be possible to control the spatial distribution of bioadhesive and nonadhesive surface areas, allowing one to control the size, shape, and biochemical state of cell cultures on a cell-by-cell basis. $^{20,21}$ However, without a thorough understanding of the properties of such microplasmas, it is not likely that they can be controlled sufficiently to produce the desired surface modifications.

Thrombus formation and embolization are significant problems for blood-contacting biomedical devices. ${ }^{22}$ Two major components of thrombi are blood platelets and the protein fibrinogen. When a surface is exposed to blood, protein adsorption is the first step in a series of events leading to thrombus formation and complement activation. ${ }^{23}$ As a result of the very rapid protein adsorption, the interaction of platelets and other blood componets with the surface may not be governed by the material directly but actually is mediated by the residing protein layer. ${ }^{24}$ In general, blood proteins with adhesive functions, ${ }^{25,26}$ such as fibronectin, fibrinogen, and von Willebrand factor, increase thrombosis, while proteins which have other functions, such as albumin, tend to decrease subsequent thromboembolic events. ${ }^{27}$ Therefore, a nonfouling polymer surface that exhibits very low absorption of platelet adhesion-simulating proteins may be useful for reducing the thromboembolic character of materials. Poly(ethylene oxide) (PEO) and PEO-like surfaces are signifi- 
cantly advantageous in preparing medical devices that require good blood compatibility, since such surfaces exhibit very low fibrinogen adsorption. ${ }^{5}$

The simplest structure that a biomedical device can have is that of a tube. This includes, but is not limited to, artificial blood vessels (vascular grafts) of various diameters as well as catheters. The use of biomaterials for vascular grafts to replace diseased human blood vessels has tremendously increased over the past 30 years. ${ }^{28,29}$ In contrast to largediameter vascular grafts (i.e., larger than $5 \mathrm{~mm}$ inside diameter) which remain excellent for more than 10 years after implantation, ${ }^{30}$ small-diameter vascular grafts ${ }^{31}$ occlude rapidly after implantation. ${ }^{32}$ Several different approaches have used plasma modification to treat the lumenal surfaces of polyethylene terephthalate ${ }^{33,34}$ (PET, Dacron), expanded polytetrafluoroethylene ${ }^{33,35}$ (ePTFE), polyethylene ${ }^{36}$ (PE), and polyurethane ${ }^{8}$ tubes. The traditional method for coating the lumenal surfaces of these polymer tubes uses plasma polymerization (PP) of a monomer gas, so as to modify the surface of the material directly or to introduce functional groups that can later be used to immobilize specific biomolecules or proteins. However, the key problem with previous approaches is the lack of the ability to control the uniformity of the coating along the length of the tubing which includes the coating thickness as well as its chemical composition. ${ }^{7}$ This is due to the inherent drawback when using PP where continuous consumption of the monomer gas and removal of the residual gases is a mandatory condition for the uniformity of the coating. ${ }^{1,7}$ To treat small diameter tubing with lengths longer than a few centimeters, the rf electrodes must be moved with respect to the tubing ${ }^{2,33,35,36}$ or the tubing must be moved continuously with respect to the electrodes. 1,7 Therefore, precise control of the speed of the electrodes or tubing, whichever is moving, is necessary for a uniform thickness of the coating along the tube. Special attention is needed to avoid deposition of an excessively thick coating, which has been found to damage the barrier characteristics of the coating. ${ }^{1,7}$ Also, when PP is used to treat the lumenal surface of polymer tubing, one end of the tubing is connected to the monomer inlet system and the other end is connected to a vacuum pump. This makes it is difficult to supply the monomer gas in the proper concentration to the location of the plasma. ${ }^{1,7}$ Increasing the pressure drop across the tubing, to supply the discharge with fresh monomer gas, will result in nonuniform plasma chemistry along the tubing. In addition, a further nonuniformity arises due to the change in the coupling of rf power to the microplasma while the electrodes are moved along the tubing or when the tubing is moved through the plasma region. ${ }^{1}$

In this work, we describe a approach for treating smalldiameter polymer tubing and we make use of two microplasma diagnostics to monitor the plasma properties during the treatment process. The microplasma, which extends along the entire length of the tubing, is created with hollow cathode (HC) electrodes ${ }^{37-39}$ which are placed in vacuum at each end of the PE tubing. This discharge configuration has many advantages compared to previous techniques. They are (1) it is an inexpensive method to create a microplasma inside small-diameter polymer tubing, (2) when the plasma is

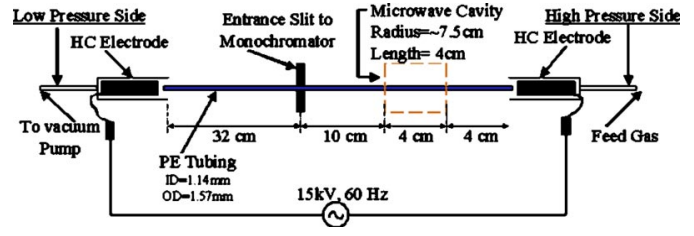

FIG. 1. Experimental configuration used to plasma treat small-diameter PE tubing and the plasma diagnostics used to monitor the properties of the plasma discharge.

created, it extends along the entire length of tubing thus eliminating the need for movable electrodes or tubing, and (3) the plasma can be created along an extended length of the tubing, up to $2 \mathrm{~m}$.

Instead of using PP, we use plasma immobilization $(\mathrm{PI}),{ }^{40-42}$ a relatively new technique, ${ }^{43,44}$ to immobilize PEO surfactants ${ }^{45,46}$ to the lumenal surface of PE tubing. We will show that immobilized PEO is useful for the reduction of adhering human platelets to the lumenal surface after exposure to blood flow in an in vitro blood circulation loop. The benefits of using this microplasma discharge together with the PI technique will give us the ability to uniformly control the surface treatment of the lumenal surface along the entire length of small-diameter polymer tubes. In addition, the use of the appropriate diagnostics gives us the ability to study the properties of microplasmas in general, as well as to find the optimal conditions for microplasma processing of biomaterials in a small volume.

\section{EXPERIMENTAL CONFIGURATION FOR MICROPLASMA TREATMENT}

To prepare for PI, microplasma surface modification was used to modify the lumenal surface of small-diameter polyethylene (PE) tubing (i.d. $1.14 \mathrm{~mm}$, o.d. $1.57 \mathrm{~mm}$ ). A diagram of the plasma discharge configuration and the diagnostics used in this work is shown in Fig. 1. The lumenalsurface modification was accomplished by placing $\mathrm{HC}$ electrodes in vacuum at each end of the polymer tubing. Two different $\mathrm{HC}$ electrode sizes were used at each end of the polymer tube. The low-pressure side had a HC electrode with an inner diameter of $8 \mathrm{~mm}$ and the high-pressure side $\mathrm{HC}$ electrode had a diameter of $6 \mathrm{~mm}$. The electrodes were powered by a $15 \mathrm{kV}$ ac transformer, which was controlled with an autotransformer in the primary of the transformer. Two plasma diagnostics (the monochromator and the microwave cavity, shown in Figs. 1 and 2) were used to monitor the uniformity of the plasma along the length of the polymer tubing by examining the light output and the plasma electron density, respectively. The tubing and the $\mathrm{HC}$ electrodes were attached to a movable table, so that the tubing can be passed through the microwave cavity and in front of the entrance slit of the monochromator simultaneously. Thermocouple gauges monitored the pressure at each end of the PE tube. The pressure drop across the tube can be controlled by pumping on both ends of the tubing and adjusting the throttle valves at each end of the tube, while inletting gas at one end. We will show that by controlling the pressure drop across the polymer tubing the uniformity of the plasma treatment can be optimized. Long plasma exposure times had to be avoided to 


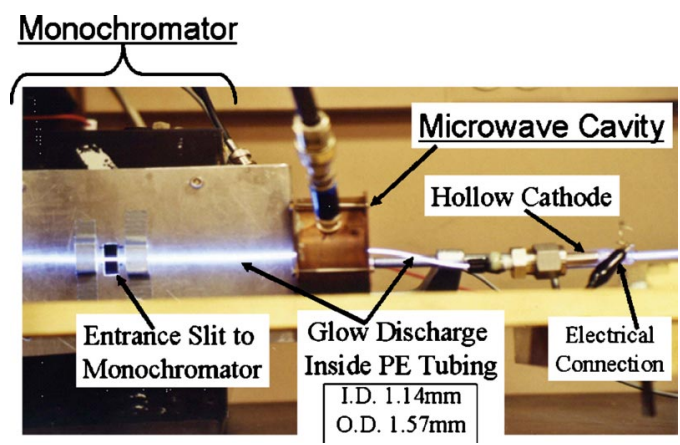

FIG. 2. (Color online) Picture of the monochromator and microwave cavity diagnostics taking measurements of an air plasma inside of a PE tubing ( i.d. $=1.14 \mathrm{~mm}$, o.d. $=1.57 \mathrm{~mm}$ ).

keep the PE tube from melting. To accomplish this, the PE tubes were exposed to a one-second plasma pulse and then the tube was allowed to cool before the next pulse took place.

\section{MICROPLASMA DIAGNOSTICS}

When a plasma is contained in a small volume or small cross-sectional area, the plasma properties such as electron density, chemical composition, temperature, spectral emission, etc., are often much different from those which is typically found in a conventional larger-volume reactor. Thus, the conditions under which microplasmas modify the surface of biomaterials will also be different. A thorough understanding of the properties of such plasmas is key to controlling the surface properties that can be obtained with microplasma discharges. This drives the necessity for noninvasive diagnostics, since immersing a probe into such plasmas is very likely to significantly perturb them from their normal operating properties. ${ }^{47}$

To determine the uniformity of the microplasma during the treatment of PE tubing, the intensity of the $3570 \AA$ nitrogen line emitted from an air plasma discharge was monitored as a function of distance along the tube. In addition, the microwave cavity diagnostic gave us an estimate of the plasma density of the microplasma in a small-diameter polytetrafluoroethylene (PTFE) polymer tube. A photograph of the monochromator and microwave cavity diagnostics used in this work is shown in Fig. 2.

\section{A. Microwave cavity measurements}

The microwave cavity method for measuring plasma density and collision frequency has been used for a long time. ${ }^{48}$ However, with the emergence of microplasmas, the use of this diagnostic has been mostly overlooked. The microwave cavity method may be an ideal diagnostic for measuring the properties of microplasmas, since a microwave cavity can be made into almost any arbitrary shape and a number of electromagnetic modes can be excited. Rightcircular cavities driven in the $\mathrm{TM}_{010}$ mode have been widely used in the microwave portion of the spectrum for plasma measurements ${ }^{49,50}$ since the 1950's. In this method, the plasma is placed in a microwave cavity, and the change of

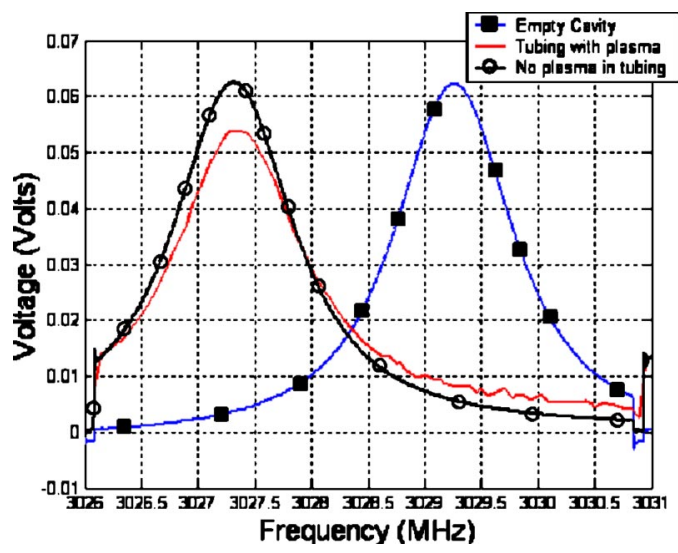

FIG. 3. (Color online) Resonant mode of the $\mathrm{TM}_{010}$ mode without the polymer tubing, with the polymer tubing, and with both the polymer tubing and plasma discharge present in side of the microwave cavity.

resonant frequency and $\mathrm{Q}$ value of the cavity are measured. ${ }^{51}$ From these measurements one can calculate the plasma density $^{50,52}$ and collision frequency. ${ }^{53}$

A copper cylindrical microwave cavity of radius $75 \mathrm{~mm}$ and height $40 \mathrm{~mm}$ was constructed that had a $\mathrm{TM}_{010}$ resonant frequency of $3029 \mathrm{MHz}$. The output of a Hewlett-Packard 83623A synthesizer-sweeper was coupled through a $\frac{1}{2}$-turn small-diameter loop placed on the inner circular surface of the cavity half-way between the ends. An identical loop placed $180^{\circ}$ around the cavity from the input loop fed a diode detector. This arrangement can thus measure the transmission through the cavity as a function of frequency. Figure 3 shows the results of three scans from 3026 to $3031 \mathrm{MHz}$. The three scans are (1) empty cavity, (2) empty PTFE tubing placed along the axis of the cavity, and (3) scan number 2 with an air plasma formed inside the tubing. PTFE tubing has a higher melting temperature than PE so it was used in the initial testing of the diagnostic. In Fig. 3, it can be seen that when no tubing is inside of the cavity, the resonant frequency of the $\mathrm{TM}_{010}$ mode is $\approx 3029.27 \mathrm{MHz}$. When the PTFE tubing (i.d.=1.14 mm, o.d. $=1.57 \mathrm{~mm}$ ) was inserted along the axis of the microwave cavity, the resonant frequency of the $\mathrm{TM}_{010}$ mode shifted down by $2.03 \mathrm{MHz}$. This is because the dielectric constant of $\mathrm{PE}$ is greater than that of air and thus the resonant frequency of the $\mathrm{TM}_{010}$ mode decreases. ${ }^{51}$ When a plasma is created inside of the tubing, since the plasma's dielectric constant is less than that of vacuum, the resonant frequency of the $\mathrm{TM}_{010}$ mode shifts up and the $Q$ of the cavity gets smaller since the plasma is lossier than vacuum. This can be seen in Fig. 3 where both the upward shift and the increase in width of the resonance (lower $Q$ ) are evident. The shift of the resonant frequency caused by the presence of the plasma is small, but it is measurable and had a value of $0.03 \mathrm{MHz}$. Initial estimates of the plasma density were found using a previously developed model, ${ }^{52}$ which gave a value of about $4.8 \times 10^{10}$ electrons $/ \mathrm{cm}^{3}$ for the plasma electron density. During this measurement the low-pressure side of the tubing was at 1.8 Torr, the high pressure side was at 2 Torr and the microwave cavity was placed $20 \mathrm{~cm}$ from the high pressure side of a $50 \mathrm{~cm}$ long PTFE tube. 


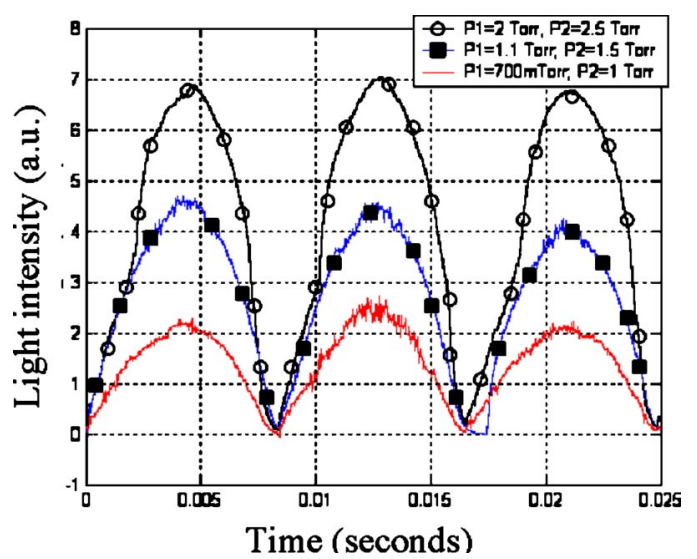

FIG. 4. (Color online) Light emitted from the $3570 \AA$ nitrogen line as a function of time for different feed pressures.

\section{B. Monochromator measurements}

Optical emission spectroscopy ${ }^{54}$ provides both the identification and monitoring of light emitting species in the microplasma during processing. Figure 4 shows the intensity of the emission from the $3570 \AA$ nitrogen line as a function of time over several ac periods, for three different fill pressures. It can be seen that as the pressure is increased, the intensity of the light emitted from the plasma also increases. Thus, if a pressure drop is created along the length of a small-diameter polymer tube, the intensity of the light emitted from the microplasma as a function of distance along the tubing should provide information about the uniformity of the discharge.

To test this hypothesis, the intensity of light emitted from the plasma that passes through the wall of the PE tubing from the $3570 \AA$ line as a function of position along the length of a $50 \mathrm{~cm}$ long polymer tubing was recorded. By changing the pressures at each end of the polymer tubing, we can control the total pressure drop across the tubing. Two conditions were chosen (1) a uniform plasma case (lowpressure side $=140 \mathrm{mTorr}$, high-pressure side $=190 \mathrm{mTorr}$ ) and (2) a nonuniform case (low-pressure side $=170 \mathrm{mTorr}$, high-pressure side $=550 \mathrm{mTorr}$ ). Thus the pressure drop was about $50 \mathrm{mTorr}$ for the uniform plasma and $380 \mathrm{mTorr}$ for the nonuniform plasma. The intensity of the $3570 \AA$ line measured as a function of distance along the tubing for the two cases is shown in Fig. 5. For the uniform plasma case, the light intensity reaches a minimum in the middle $20 \mathrm{~cm}$ of

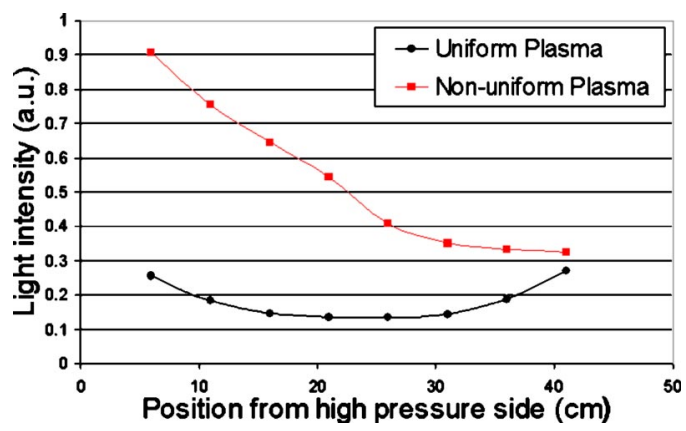

FIG. 5. (Color online) Light emitted from the $3570 \AA$ nitrogen line as a function of position along the polymer tubing for two plasma conditions, a uniform and a nonuniform air-microplasma discharge. the tubing and increases at each end of the tubing. We believe that this is due to the fact that the $\mathrm{HC}$ electrodes are at each end of the tube, and thus the resulting electric fields and therefore the plasma is more intense at each end. However, when the pressure drop across the tubing is increased to $380 \mathrm{mTorr}$ (nonuniform case) the light intensity measured along the tubing has a maximum at the high-pressure end and then it decays monotonically toward the low-pressure end of the tubing before reaching a constant level just before the $\mathrm{HC}$ electrode is reached. We conclude that the light emitted from the microplasma can become nonuniform along the tubing due to two factors, (1) if a large pressure drop is created across the tubing and (2) proximity to the electrodes that create the plasma. It is important to determine whether the light intensity measured as a function of position along the length of the polymer tubing can be used to predict the uniformity of the surface treatment created by a uniform or nonuniform plasma discharge. To test this hypothesis, the treatment effectiveness along the lumenal surface of the tubing was evaluated using the capillary-rise method, which can be directly related to the contact angle.

\section{CAPILLARY-RISE METHOD}

Effectiveness of the plasma treatment on the lumenal surface of the PE tubing was evaluated using the capillaryrise (CR) method. ${ }^{8}$ Use of the CR method allows us to monitor the uniformity of the contact angle inside the PE tubing, which is believed to be very dependent on the surface properties along the tubing. The $\mathrm{CR}$ measurements of the PE tubing were obtained by cutting the $50 \mathrm{~cm}$ long plasmatreated tubing into ten sections of $5 \mathrm{~cm}$ length. Each section was then dipped in a beaker of de-ionized (DI) water such that at least $4 \mathrm{~cm}$ of the section was submerged. ${ }^{8}$ Then the tube was slowly withdrawn from the water, and the height of the water column supported by the surface tension on the lumenal surface was measured. The contact angle of the lumenal surface is related to the height of the column of liquid by the expression which is obtained from the Young-Laplace equation $^{55}$

$$
h=\frac{2 s \cos \theta}{n g r},
$$

where $h$ is the height of the liquid (it can be negative if the liquid falls below the surface of the water outside the tubing), $s$ is the surface tension of the liquid, $r$ is the radius of curvature of the tubing, $n$ is the density of the liquid, $g$ is the gravitational constant, and $\theta$ is the contact angle of the liquid on the capillary surface. The lumenal surface of the PE tubing (i.d. $=1.14 \mathrm{~mm}$, o.d. $=1.57 \mathrm{~mm}$ ), in its original state from the vendor, exhibited a negative capillary rise of $6 \mathrm{~mm}$, which corresponds to a contact angle $\theta$ of $\approx 92^{\circ}$, which is close to values ${ }^{44}$ that have been previously reported for flat untreated PE surfaces. To determine whether a change in pressure drop along the tubing, during plasma treatment, affects the CR measurements, the total pressure drop along the tubing was changed. The same plasma conditions used for the monochromator uniformity measurements were also used here, which were (1) uniform plasma (low-pressure side 


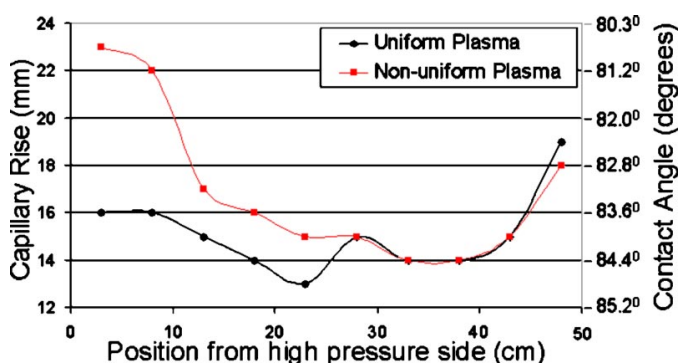

FIG. 6. (Color online) The capillary rise measurements taken as a function of position along two PE tubes exposed to two different plasma conditions, a uniform and a nonuniform air-microplasma discharge.

$=140$ mTorr, high-pressure side $=190 \mathrm{mTorr}$ ) and (2) nonuniform plasma (low-pressure side $=170 \mathrm{mTorr}$, highpressure side $=550 \mathrm{mTorr})$. For the uniform plasma case, the PE tube was exposed for two one-second pulses, while for the nonuniform case, the tube was exposed for three onesecond pulses. An air plasma was used to treat the tubing in both cases. The results of the CR measurements, for the two cases, as a function of distance along the plasma treated tubing is shown in Fig. 6. The left-hand scale shows the CR rise of the DI water and the corresponding contact angle is shown on the right-hand scale. It can be seen that for both cases, there is a minimum CR near the middle of the tubing. The $\mathrm{CR}$ increases near the ends of the tubing. Again, similarly to the monochromator measurements, we believe that this is due to the fact that the electrodes that create the plasma are located at the ends of the tubing, where the plasma is more intense. For the nonuniform case, the CR measured along the tubing has a maximum at the high-pressure end and then it drops toward the low-pressure side. This is very similar to the monochromator measurements under the same conditions. However, unlike the monochromator measurements, which flatten out at the low-pressure end, the CR keeps increasing toward the low-pressure side. We conclude that the $\mathrm{CR}$ measurements become more nonuniform along the tubing as a result of two factors, (1) when the pressure drop along the tubing is increased and (2) at points close to the electrodes.

Thus, we have shown that the intensity of light emitted from the microplasma as a function of position can be related to the uniformity of the plasma along the treated tubing. In addition, light intensity can be related to the CR along the tubing under similar plasma conditions. It is also important to note that, for five or more one-second pulses in an airplasma, for both the uniform and nonuniform plasma, the $\mathrm{CR}$ reaches a maxiumum of $27 \mathrm{~mm}\left(\theta \sim 79^{\circ}\right.$ contact angle), which is uniform along the whole length of the PE tubing. Therefore the uniformity of the treatment can be measured with the CR method for extended treatment times whether or not the plasma is uniform. However, it is expected that without careful optimization of the plasma, the ends of the tubing where the plasma is more intense may be degraded before the lumenal surface near the middle of the tube is fully treated. This can become more of a problem as the length of the tube is increased.

\section{COATING AND IMMOBILIZATION OF PEO SURFACTANTS ONTO PE TUBING}

Using the plasma immobilization (PI) technique, it has been previously shown that when PEO chains are immobilized on a PE surface, the adsorption of proteins such as fibrinogen can be reduced significantly. ${ }^{43-45}$ Such a nonfouling polymer surface was obtained by exposing a flat PE substrate, which was precoated with a PEO surfactant, to a short, low-power Ar plasma treatment. In this section, we describe how to apply this technique to immobilize a PEO surfactant to the lumenal surface of small-diameter PE tubing.

The PI technique consists of a two-step process. ${ }^{56}$ First, a surfactant is coated on the surface to be modified and then it is exposed to an inert gas plasma. During the plasma exposure, crosslinking between the surfactant and the polymer substrate may occur, thus covalently bonding part of the precoated surfactant to the lumenal surface of the tubing by the "CASING" method ${ }^{43-45}$ (CASING—crosslinking by active species of inert gases). Since an inert gas is used, there is no significant change in gas-phase plasma chemistry, since argon is not consumed by the surfactant or the substrate during plasma exposure. ${ }^{45}$ The inert-gas plasma acts only as a crosslinking agent through the bombardment of energetic electrons, ions, and photons. This technique is ideal for treating the lumenal surface of small-diameter polymer tubing, since the consumption of gas in negligible, making it easier to control the uniformity of the microplasma treatment. There is one important step that must be taken before exposing the surface to plasma: the surfactant coating must wet the surface to be modified otherwise there will be no surfactant at the surface during the CASING step.

To improve wetting of the PE surface by a $10 \% \mathrm{w} / \mathrm{v}$ aqueous PEO surfactant (Brij 98) solution, the hydrophilicity of the lumenal surface of the PE tubing was increased. This was accomplished by introducing polar functional groups at the surface by means of plasma treatment. ${ }^{56}$ The inside of the PE tubing was exposed to four one-second pulses of a uniform air plasma. The tubing was then removed from the reactor, and the PEO surfactant was injected into the PE tubing and kept in contact with the lumenal surface for $30 \mathrm{~min}$ at room temperature (RT). Subsequently, the surfactant was withdrawn, and the tube was air dried at RT for 30 min while the solvent evaporated, leaving behind a thin coating of PEO polymer on the lumenal surface. The precoated tube was then reconnected to the vacuum system and exposed to an argon plasma, under uniform-plasma conditions, for a total of ten one-second pulses. After the final plasma treatment, the tube was flushed with DI water for 5 min and then soaked in fresh DI water before blood compatibility testing was undertaken.

\section{BLOOD-COMPATIBILITY TESTING}

To determine whether the PEO that has been plasmaimmobilized on the lumenal surface of PE tubing can reduce the adhesion of human blood platelets, an in vitro bloodcirculation loop was constructed. A diagram of the in vitro loop is shown in Fig. 7. For this experiment, $20 \mathrm{ml}$ of blood was obtained by a standard venipuncture technique from a 


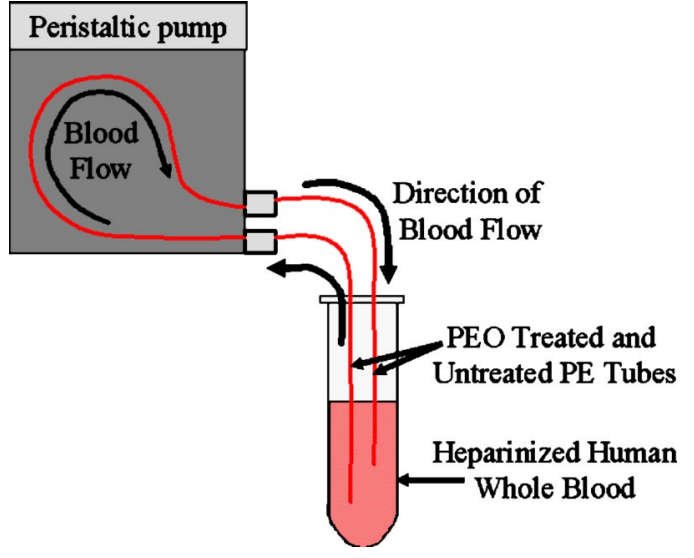

FIG. 7. (Color online) Diagram of the in vitro flow loop which circulates heparinized whole human blood simultaneously through both a treated and untreated PE tubing.

healthy human donor. The blood was heparinized and stored at $37{ }^{\circ} \mathrm{C}$ for $20 \mathrm{~min}$ until being placed in the bloodcirculation loop reservoir. As shown in Fig. 7, a peristaltic pump circulated blood through two separate $26 \mathrm{~cm}$ long PE tubes, one of which was PEO plasma immobilized, while the other remained untreated. Blood was circulated for $1 \mathrm{~h}$ at $37{ }^{\circ} \mathrm{C}$ at a flow rate of $0.75 \mathrm{ml} / \mathrm{min}$. Initially, the blood-flow direction from the reservoir passed through the treated tubing, then through the peristaltic pump, and finally through the untreated tubing before returning to the reservoir. After $30 \mathrm{~min}$, the flow direction was reversed. At the conclusion of this process, the tubing was rinsed with phosphate buffer solution (PBS) that was circulated through each of the two pieces of tubing for $5 \mathrm{~min}$ in each direction, at the same temperature and flow rate as the blood. Then, a $1.25 \% \mathrm{v} / \mathrm{v}$ solution of glutaraldehyde ( $25 \%$ solution) and $1 \% \mathrm{w} / \mathrm{v}$ of tannic acid in PBS was flushed through both of the tubes for 15 min in each direction at RT, with the same flow rate as above. The purpose of the glutaraldehyde solution is to fix the platelets and cells that may be attached to the lumenal surface of the PE tubes. The tannic acid improves scaning electron microscopy imaging. After the fixation step, the tubes were rinsed with PBS at RT for another $5 \mathrm{~min}$ in each direction. The fixed cells and platelets were then dehydrated by flushing the tubes with increasing concentrations of ethanol from $50 \%$ to $95 \%$ in steps of $5 \%$ for two-minute exposures at each concentration. This was also done at RT with the same flow rate as above.

At the conclusion of this step, each of the two tubes was cut into $4 \mathrm{~cm}$ long sections. Then, $1 \mathrm{~cm}$ subsections were cut from the middle of each of the $4 \mathrm{~cm}$ sections. The resulting six samples from both tubes were then prepared for SEM imaging. Figures 8 and 9 show SEM images of both the untreated and PEO-treated tubes after blood exposure. To facilitate comparison, and to utilize the uniformly treated region of the tubing, SEM images shown in Figs. 8 and 9 were obtained at the same point, near the middle of both the treated and untreatd tubes.

It is important to note, when individual platelets attach to a surface, they spread and undergo cytoskeletal reorganization. $^{22,57}$ When the spreading platelets come in

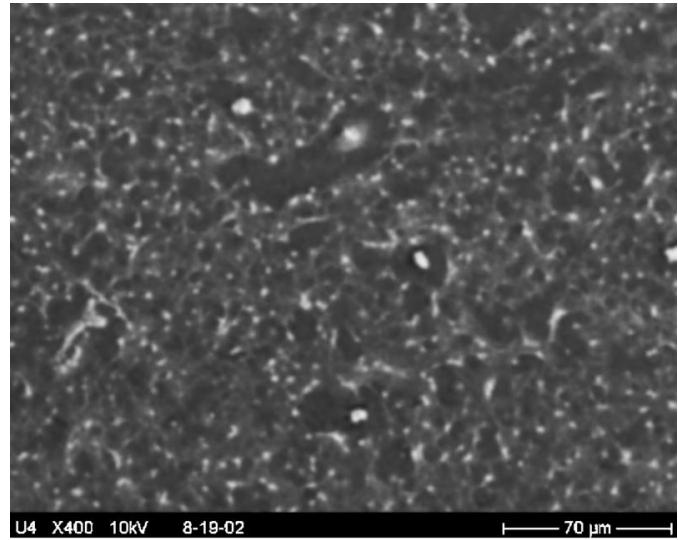

(a)

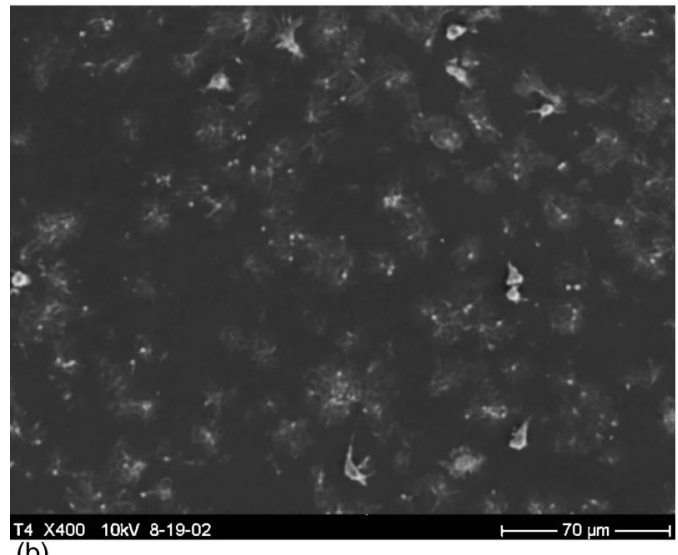

(b)

FIG. 8. SEM images of the lumenal surface of (a) untreated and (b) PEO treated PE tubing after exposure to blood flow at a rate of $0.75 \mathrm{ml} / \mathrm{min}$ at $37{ }^{\circ} \mathrm{C}$ for $1 \mathrm{~h}$.

contact with each other, they act in a coordinated fashion and form a base for thrombus initiation and growth. ${ }^{22}$ The final steps in the coagulation cascade include formation of thrombin, which converts fibrinogen into fibrin monomers, from which a fibrin network is produced. ${ }^{24,57}$ All of these conditions can be seen in both Figs. 8 and 9 .

Figures 8(a) and 8(b) show the adhering blood components (mostly platelets) on the lumenal surface of the untreated and PEO-treated tubes, respectivly. It can be seen that the number of platelets adhering to the PEO-treated tubing is much less than the number adhering to the untreated PE tube. In Fig. 8(a), it can be seen that numerous microthrombi have formed on the lumenal surface of the untreated PE tube after $1 \mathrm{~h}$ of exposure to heparinized blood. In Fig. 8(b), it can be seen that there are a relatively few number of spreading platelets on the PEO-treated tube, and even fewer bases for microthrombi. Under higher magnification, shown in Figs. 9(a) and 9(b), it can be seen that the fibrin network is far more complex on the untreated tubing compared to the treated tubing.

The SEM images of the other locations along the untreated PE tube show that the density of platelets adhering to the lumenal surface is not uniform. It appears that this density distribution of adhering platelets along the untreated PE tube depends on the proximity to the peristaltic pump powering the blood-circulation loop. The density of platelets ad- 


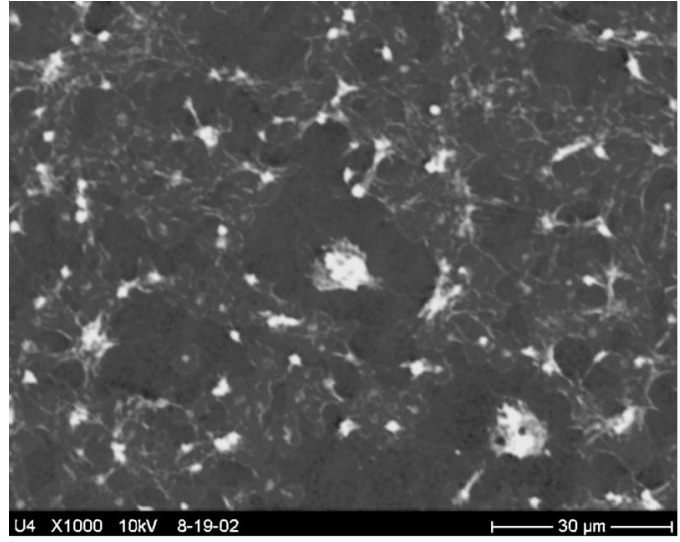

(a)

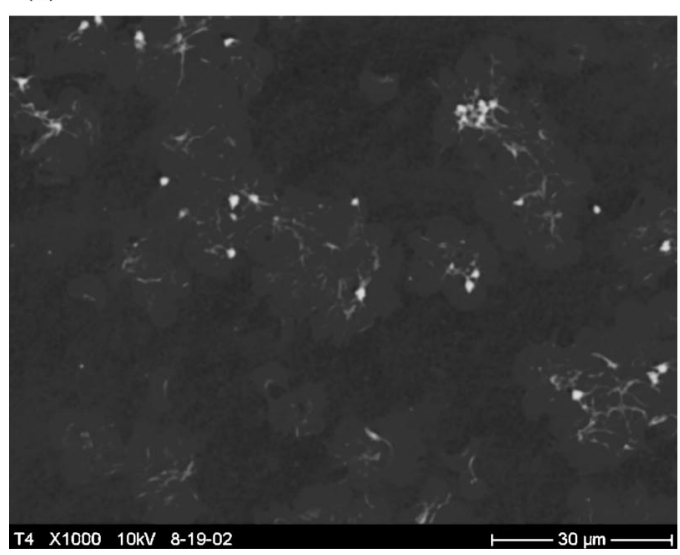

(b)

FIG. 9. SEM images of the lumenal surface of (a) untreated and (b) PEO treated PE tubing after exposure to blood flow under higher magnification than Fig. 8.

hering to the untreated PE tube increases as we move away from the peristaltic pump and closer to the blood reservoir. Since the untreated tubing is expected to have uniform lumenal surface properties, it is likely that the nonuniformity of the platelet density may be due to the blood-flow properties of the in vitro blood-circulation system used in this work. However, it is interesting to note that the SEM images taken at the other locations along the PEO-treated tubing showed similar results to these shown in Figs. 8(b) and 9(b), that is, a uniform density of adhering blood platelets was found along the PEO-treated tube. Thus, we conclude that the number of adhering platelets on the lumenal surface of PE tubing can be reduced by immobilizing a PEO surfactant to the lumenal surface of the tubing using a microplasma discharge.

\section{SUMMARY}

Two microplasma diagnostics were used to monitor the plasma properties. A microplasma was created with $\mathrm{HC}$ electrodes which are placed at each end of the tube. It was shown that by suitably controlling the pressure drop along the tube, the degree of plasma uniformity as a function of distance along the tubing can be optimized. Emitted light from the plasma was fed into a monochromator at various positions along the PE tube to measure the uniformity. The monochromator measurements were then related to the capillary rise measured along the length of the polymer tubing for uniform and nonuniform plasma conditions. It was shown that a nonuniform plasma treatment can be due to two factors (1) a pressure drop created across the PE tubing and (2) proximity to the electrodes. A microwave-cavity diagnostic was used to measure the density of an air-microplasma-a value of 4.8 $\times 10^{10}$ electrons $/ \mathrm{cm}^{3}$ was obtained.

PI was used to immobilize a PEO surfactant to the lumenal surface of small-diameter PE tubing. It was seen that the use of this method to immobilize PEO surfactants to the lumenal surface of PE tubing showed a significant decrease in the number of platelets adhering to the lumenal surface compared with untreated PE tubing.

${ }^{1}$ Y. Matsuzawa and H. Yasuda, Org. Coat. Appl. Polym. Sci. Proc. 47, 397 (1982).

${ }^{2}$ K. Hatada and H. Kobayahi, Coat. Appl. Polym. Sci. Proc. 47, 391 (1982).

${ }^{3}$ M. Sheu, M. S. Wagner, D. G. Castner, B. D. Ratner, and T. A. Horbett, Langmuir 19, 1692 (2003).

${ }^{4}$ M. Sheu, L. Martinson, M. S. Wagner, D. G. Castner, B. D. Ratner, and T. A. Horbett, J. Biomater. Sci., Polym. Ed. 13, 367 (2002).

${ }^{5}$ M. Sheu, Y. V. Pan, M. S. Wagner, K. D. Hauch, D. G. Castner, B. D. Ratner, and T. A. Horbett, J. Biomater. Sci., Polym. Ed. 12, 961 (2001).

${ }^{6}$ Polymeric Biomaterials, edited by S. Dumitriu (Marcel Dekker, Inc., New York, 1994).

${ }^{7}$ Y. Matsuzawa and H. Yasuda, J. Appl. Polym. Sci. 38, 65 (1984).

${ }^{8}$ P. V. Narayanan, J. Biomater. Sci., Polym. Ed. 6, 181 (1994).

${ }^{9}$ T. Ichiki, Y. Sugiyama, R. Taura, T. Koidesawa, and Y. Horiike, Thin Solid Films 435, 62 (2003).

${ }^{10}$ F. Iza and J. A. Hopwood, IEEE Trans. Plasma Sci. 31, 782 (2003).

${ }^{11}$ A. M. Bilgic, U. Engel, E. Voges, M. Kuckelheim, and J. A. C. Broekaert, Plasma Sources Sci. Technol. 9, 1 (2000).

${ }^{12}$ A. M. Bilgic, E. Voges, U. Engel, and J. A. C. Broekaert, J. Anal. At. Spectrom. 15, 579 (2000).

${ }^{13}$ Y. B. Guo and F. C. N. Hong, Appl. Phys. Lett. 82, 337 (2003).

${ }^{14}$ H. Yoshiki, A. Oki, H. Ogawa and Y. Horiike, J. Vac. Sci. Technol. A 20, 24 (2002).

${ }^{15}$ N. Gherardi, G. Gouda, E. Gat, A. Ricard, and F. Massines, Plasma Sources Sci. Technol. 9, 340 (2000).

${ }^{16}$ J. A. Hopwood, J. Microelectromech. Syst. 9, 309 (2000).

${ }^{17}$ C. G. Wilson, Y. B. Gianchandani, R. R. Arslanbekov, V. Kolobov, and A. E. Wendt, J. Appl. Phys. 94, 2845 (2003).

${ }^{18}$ T. Ono, D. Y. Sim and M. Esashi, J. Micromech. Microeng. 10, 445 (2000).

${ }^{19}$ L. D. Biborosch, O. Bilwatsch, S. Ish-Shalom, E. Dewald, U. Ernst and K. Frank, Appl. Phys. Lett. 75, 3926 (1999).

${ }^{20}$ Y. V. Pan, T. C. McDevitt, T. K. Kim, D. Leach-Scampavia, P. S. Stayton, D. D. Denton, and B. D. Ratner, Plas. and Polym. 7, 171 (2002).

${ }^{21}$ J. A. Lundqvist, F. Sahlin, M. A. I. Aberg, A. Stromberg, P. S. Eriksson, and O. Orwar, Proc. Natl. Acad. Sci. U.S.A. 95, 10356 (1998).

${ }^{22}$ Q. J. Lai, S. L. Cooper, and R. M. Albrecht, Procedings of the XIIth International Congress for Electron Microscopy, 849 (1990)

${ }^{23}$ A. Kidane and K. Park, J. Biomed. Mater. Res. 48, 640 (1999).

${ }^{24}$ E. Brynda, M. Houska, M. Jirouskova, and J. E. Dyr, J. Biomed. Mater. Res. 31, 249 (2000).

${ }^{25}$ L. K. Lambrecht, B. R. Young, R. E. Stafford, K. Park, R. M. Albrecht, D. F. Mosher, and S. L. Cooper, Thromb. Res. 41, 99 (1986).

${ }^{26}$ J. M. Grunkemeier, W. B. Tsai, and T. A. Horbett, J. Biomater. Sci., Polym. Ed. 12, 1 (2001).

${ }^{27}$ S. L. Goodman, S. L. Cooper and R. M. Albrecht, J. Biomater. Sci., Polym. Ed. 2, 147 (1991).

${ }^{28}$ F. R. Pu, R. L. Williams, T. K. Markkula, and J. A. Hunt, Biomaterials 23, 2411 (2002).

${ }^{29}$ K. N. Kader, J. M. Sweany, and R. V. Bellamkonda, J. Biomed. Mater. Res. 60, 405 (2002).

${ }^{30}$ J. H. Lee, S. J. Lee, G. Khang, and H. B. Lee, J. Colloid Interface Sci. 230, 84 (2000).

${ }^{31}$ L. Xue and H. P. Greisler, J. Vasc. Surg. 37, 472 (2003).

${ }^{32}$ G. W. Bos, A. A. Poot, T. Beugeling, W. G. van Aken, and J. Feijen, Arch. Physiol. Biochem. 106, 110 (1998). 
${ }^{33}$ T. Chandy, G. S. Das, R. F. Wilson, and G. H. R. Rao, Biomaterials 21, 699 (2000).

${ }^{34}$ D. Kiaei, A. S. Hoffman, B. D. Ratner, and T. A. Horbett, J. Appl. Polym. Sci. 42, 269 (1988).

${ }^{35}$ D. Y. Tseng and E. R. Edelman, J. Biomed. Mater. Res. 42, 188 (1998).

${ }^{36}$ Jui-Che Lin and S. L. Cooper, Biomaterials 16, 1017 (1995).

${ }^{37}$ K. H. Schoenbach, M. Moselhy, W. Shi, and R. Bentley, J. Vac. Sci. Technol. A 21, 1260 (2003).

${ }^{38}$ K. H. Schoenbach, R. Verhappen, T. Tessnow, F. E. Peterkin, and W. W. Byszewski, Appl. Phys. Lett. 68, 13 (1996).

${ }^{39}$ S. J. Park and J. G. Eden, Appl. Phys. Lett. 81, 4127 (2002).

${ }^{40}$ J. P. Lens, J. G. Terlingen, G. H. Engbers, and J. Feijen, J. Biomater. Sci., Polym. Ed. 9, 357 (1998).

${ }^{41}$ J. P. Lens, J. G. Terlingen, G. H. Engbers, and J. Feijen, Polymer 39, 3437 (1998).

${ }^{42}$ J. P. Lens, J. G. Terlingen, G. H. Engbers, and J. Feijen, Langmuir 13, 7052 (1997).

${ }^{43}$ M. S. Sheu, A. S. Hoffman, and J. Feijen, J. Adhes. Sci. Technol. 6, 995 (1992).

${ }^{44}$ M. S. Sheu, A. S. Hoffman, J. G. A. Terlingen, and J. Feijen, J. Clin
Mater. 13, 41 (1993).

${ }^{45}$ M. S. Sheu, A. S. Hoffman, B. D. Ratner, J. Feijen, and J. M. Harris, J. Adhes. Sci. Technol. 7, 1065 (1993).

${ }^{46}$ J. P. Lens, P. H. Harmsen, E. M. Ter Schegget, J. A. Terlingen, G. H. Engbers, and J. Feijen, J. Biomater. Sci., Polym. Ed. 8, 963 (1997).

${ }^{47}$ O. B. Minayeva and J. Hopwood, J. Appl. Phys. 94, 2821 (2003).

${ }^{48}$ B. Agdur and B. Enander, J. Appl. Phys. 33, 575 (1962).

${ }^{49}$ K. B. Persson, Phys. Rev. 106, 191 (1957).

${ }^{50}$ K. I. Thomassen, J. Appl. Phys. 36, 3642 (1965).

${ }^{51} \mathrm{~S}$. Li, C. Akyel, and R. G. Bosisio, IEEE Trans. Microwave Theory Tech. 29, 1041 (1981)

${ }^{52}$ J. L. Shohet and A. J. Hatch, J. Appl. Phys. 41, 2610 (1970).

${ }^{53}$ L. Gould and S. C. Brown, J. Appl. Phys. 24, 1053 (1953).

${ }^{54}$ Plasma Deposition, Treatment, and Etching of Polymers, edited by R. d'Agostino (Academic Press, Inc., 1990).

${ }^{55}$ A. W. Adamson and A. P. Gast, Physical Chemistry of Surfaces (Wiley, Inc., 1997).

${ }^{56}$ J. P. Lens, J. G. Terlingen, G. H. M. Engbers, and J. Feijen, Langmuir 14, 3214 (1998).

${ }^{57}$ S. L. Goodman and R. M. Albrecht, Scanning Microsc. 1, 727 (1987). 